\title{
SEPIA: simulation-based evaluation of prioritization algorithms
}

\author{
Kimberly Almaraz ${ }^{\dagger}$, Tyler Jang ${ }^{\dagger}$, McKenna Lewis $^{\dagger}$, Titan $\mathrm{Ngo}^{\dagger},{ }^{\dagger}$ Miranda Song ${ }^{\dagger}$ and Niema Moshiri* (i)
}

\begin{abstract}
Background: The ability to prioritize people living with HIV (PLWH) by risk of future transmissions could aid public health officials in optimizing epidemiological intervention. While methods exist to perform such prioritization based on molecular data, their effectiveness and accuracy are poorly understood, and it is unclear how one can directly compare the accuracy of different methods. We introduce SEPIA (Simulation-based Evaluation of Prloritization Algorithms), a novel simulation-based framework for determining the effectiveness of prioritization algorithms. SEPIA expands upon prior related work by defining novel metrics of effectiveness with which to compare prioritization techniques, as well as by creating a simulation-based tool with which to perform such effectiveness comparisons. Under several metrics of effectiveness that we propose, we compare two existing prioritization approaches: one phylogenetic (ProACT) and one distance-based (growth of HIV-TRACE transmission clusters).
\end{abstract}

Results: Using all proposed metrics, ProACT consistently slightly outperformed the transmission cluster growth approach. However, both methods consistently performed just marginally better than random, suggesting that there is significant room for improvement in prioritization tools.

Conclusion: We hope that, by providing ways to quantify the effectiveness of prioritization methods in simulation, SEPIA will aid researchers in developing novel risk prioritization tools for PLWH.

Keywords: SEPIA, HIV, Prioritization, Metrics, Simulation-based evaluation, FAVITES, Phylogenetic

\section{Background}

Molecular data gathered on human immunodeficiency virus (HIV) is useful for understanding the systems of epidemic spread of HIV. Such understanding can better allow us to intervene and treat high-risk groups of individuals. Methods of epidemic intervention include treatments such as antiretroviral therapy (ART) and awareness programs [1]. Adherence to ART can cause viral suppression in people living with HIV (PLWH) and significantly reduces their risk of transmission, making ART distribution a potentially effective approach to

*Correspondence: niema@ucsd.edu

†Equal contributor: Kimberly Almaraz, Tyler Jang, McKenna Lewis, Titan Ngo and Miranda Song

Department of Computer Science and Engineering, University

of California San Diego, 9500 Gilman Drive, La Jolla, CA 92093, USA combating the spread of HIV. However, a major issue for public health officials is how to allocate the limited amount of available resources.

In many parts of the world, when testing and treating PLWH, it has become standard practice to record various metadata on the patients, including viral genomic sequences (often of the $p o l$ and gag regions). This information is often used to determine groups of individuals with high risk of future transmission, which can help public health officials better allocate limited resources [2]. The prioritization of PLWH can be explored through a computational framework: given a list of individuals along with metadata and viral sequences, order the individuals in descending order of inferred risk of future transmission.

Molecular epidemiology provides a natural framework for prioritizing individuals from viral sequence 
data. Currently, the standard approach is to use HIVTRACE [3] to infer transmission clusters based on pairwise distances between sequences, monitor the growth of the transmission clusters over time, and prioritize individuals in descending order of transmission cluster growth. ProACT [4], on the other hand, is a prioritization approach that utilizes properties of a phylogeny inferred from the viral sequences.

The following questions naturally arise: how well does a given prioritization method perform, and which method is superior in specific contexts? With real-world data, the ground truth of who transmitted to whom is typically unavailable or error-prone. Further, even with a known transmission history, it is unclear how to quantify effectiveness: do we count the number of transmissions from a single individual, or the total number of transmissions in a transmission chain seeded from a single individual, or perhaps we are interested in properties of the underlying contact network (e.g. individuals with large numbers of social contacts)? Thus, it is unclear how to quantitatively assess the performance of different prioritization methods.

To address this open problem, we introduce SEPIA (Simulation-based Evaluation of PrIoritization Algorithms), a novel simulation-based framework for measuring the effectiveness of prioritization algorithms. Previously, in Moshiri et al. (2021) [4], ProACT and HIVTRACE were compared with respect to effectiveness, but the comparisons were limited to a simulated epidemic dataset modeling the San Diego HIV epidemic between 2005 and 2014. Like this prior work, SEPIA utilizes simulated epidemic data, such as those generated by FAVITES [5] or PANGEA.HIV.sim [6], to define a ground truth with which prioritization methods can be directly compared. However, SEPIA expands upon this prior work by generalizing the task of prioritization effectiveness comparison and further exploring the mathematical meaning of "effectiveness" by defining 6 metrics of effectiveness, each inspired by properties of epidemics that are inherently of interest to public health officials for intervention. Specifically, the user runs a prioritization method on a simulated dataset; then, given the prioritization and the simulated dataset, SEPIA will measure the effectiveness of the prioritization using the metrics defined below.

\section{Implementation}

Given a prioritization, SEPIA computes an effectiveness score according to one of the following metrics:

- Metric 1: Direct Transmissions This metric aims to quantify the direct impact of each individual $u$ on the spread of the virus within a population by counting the total number of individuals to whom $u$ directly transmitted.

- Metric 2: Transmission Rate This metric aims to quantify the rate of transmission of each individual $u$, giving higher values to those who transmitted most frequently and most recently. We produce a step function representing the number of transmissions from individual $u$ over time, and we measure the slope of a regression line inferred from the step graph (Fig. 1).

- Metric 3: Indirect Transmissions This metric expands on Metric 1 to quantify an individual's broader impact on the community. For an individual $u$, we count the number of individuals who were infected by somebody who was infected by $u$.

- Metric 4: Direct and Indirect Transmissions This is the sum of Metrics 1 and 3.

- Metric 5: Number of Contacts This metric measures each individual's total number of contacts in the underlying contact network.

- Metric 6: Number of Contacts and Transmissions This is the sum of Metrics 1 and 5.

Given a prioritization of $n$ individuals and the simulated data from which the prioritization was produced, for a given selected metric, SEPIA will compute a value for each individual in the prioritization. To compute a score comparing the user's prioritization to the theoretical optimum, SEPIA computes the Kendall Tau-b rank correlation coefficient [7] between the list of ordered metric values and the descending list of integers from $n$ to 1 (Fig. 2). The resulting Tau-b score ranges from 1 (perfectly correlated with optimal ordering) to 0 (no better than random ordering) to -1 (perfectly anticorrelated with optimal ordering).

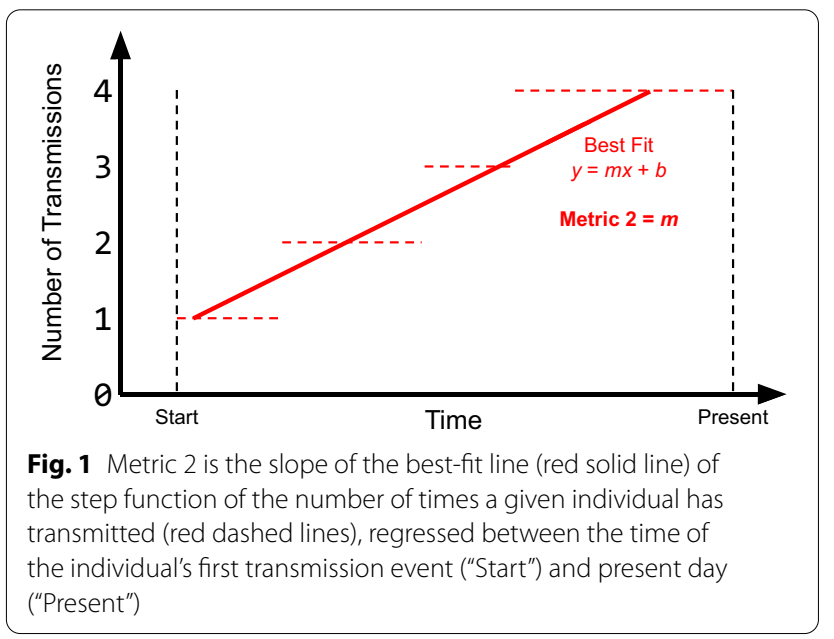



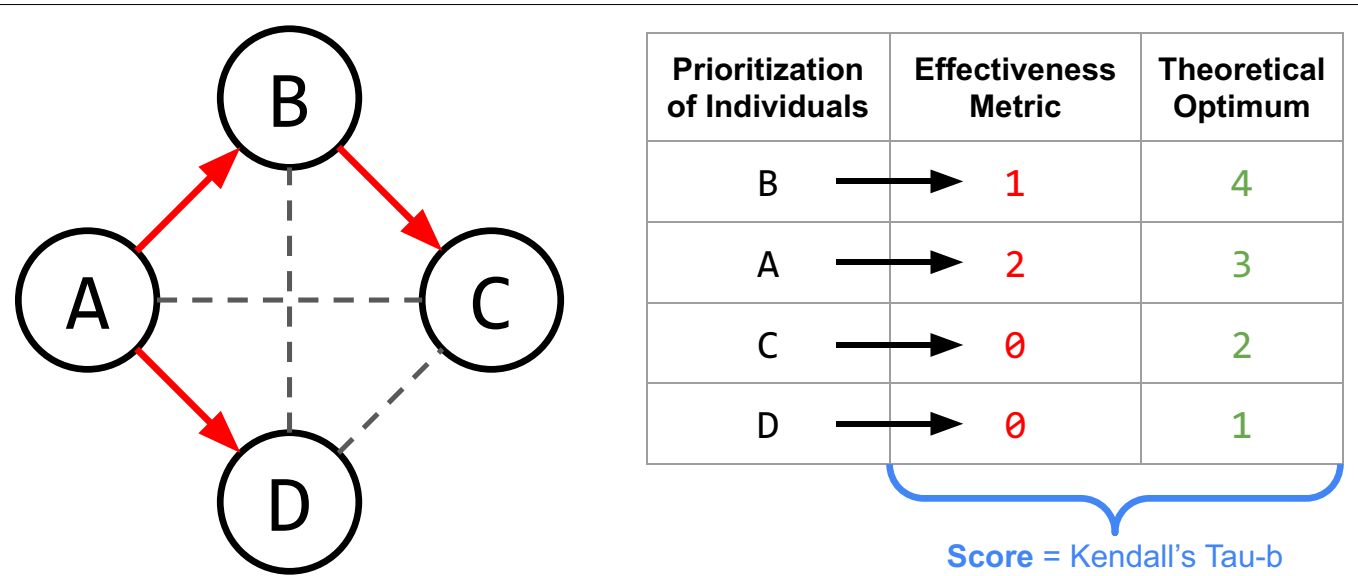

Fig. 2 Given simulated epidemic data and a prioritization of the individuals in the simulated epidemic, SEPIA computes the user-selected effectiveness metric for each person in the prioritization. Then, to construct an overall effectiveness score for this prioritization, SEPIA computes the Kendall Tau-b correlation coefficient between the ordered list of effectiveness values and the theoretical optimum

We used SEPIA to compare the effectiveness of two molecular epidemiological prioritization methods. One approach is to use HIV-TRACE to infer transmission clusters from pairwise distances of viral sequences, monitor the growth of the transmission clusters over time, and then to prioritize individuals in descending order of transmission cluster growth. The other approach is ProACT [4], a method that utilizes properties of a phylogeny inferred from the viral sequences. We used a simulated dataset produced by FAVITES to emulate the HIV pandemic in San Diego between 2005 and 2014 [5]. The simulated datasets vary the expected degree in the contact network $\left(E_{d}\right)$, the rate at which individuals begin ART $\left(\lambda_{+}\right)$, and the rate at which individuals stop adhering to ART $\left(\lambda_{-}\right)$.

\section{Results}

As can be seen in Fig. 3, ProACT consistently outperformed HIV-TRACE transmission cluster growth using all metrics on all simulation conditions. However, both tools consistently had Tau-b scores marginally higher than 0 , implying that they are performing only marginally better than a random ordering. As the rate of starting ART $\left(\lambda_{+}\right)$increases, the rate of stopping ART $\left(\lambda_{-}\right)$increases, and the expected degree $\left(E_{d}\right)$ increases (i.e., as the outbreak spreads more quickly), ProACT's performance with respect to metrics 5 and 6 seems to increase slightly. Otherwise, both ProACT and HIV-TRACE transmission cluster growth perform consistently across experimental conditions.

\section{Discussion}

Across all defined metrics and all considered simulation conditions, ProACT consistently outperformed prioritization by HIV-TRACE transmission cluster growth. However, both approaches consistently performed just marginally better than a random ordering, implying that there is room for significant improvement in the realm of HIV prioritization.

\section{Conclusions}

It must be noted that, while we aimed to provide generalized results by varying key simulation parameters, a key limitation of this study is that the simulated epidemics are specifically modeled after the HIV epidemic in San Diego between 2005 and 2014. In practice, molecular epidemiologists will need to assess prioritization techniques using simulated datasets representative of the pathogens and communities in which they are specifically interested.

Further, the 6 metrics we have implemented are by no means exhaustive, but rather, they are simply natural metrics of interest to public health officials. SEPIA is expandable, and we leave the implementation of novel metrics of effectiveness for future works.

We hope that SEPIA will enable researchers to quantify and assess the effectiveness of different prioritization approaches in order to select the best existing prioritization method for their communities, develop new prioritization methods that improve upon existing ones, and, ultimately, maximize the impact of the limited resources available to public health officials. 


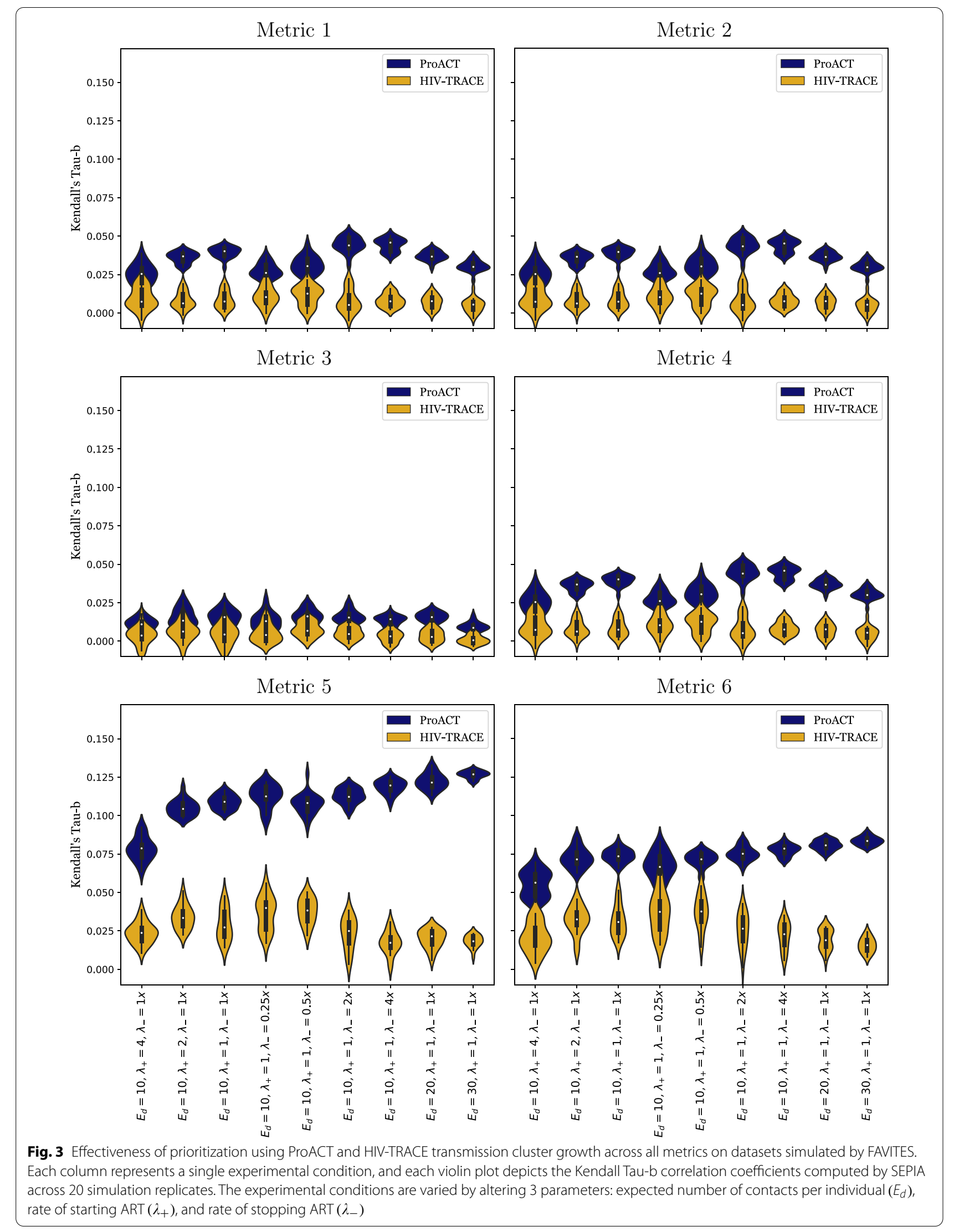




\section{Availability and requirements}

- Project name: SEPIA

- Project home page: https://github.com/Niema-Lab/ SEPIA

- Operating system(s): Platform independent

- Programming language: Python

- Other requirements: SciPy

- License: GNU GPL v3.0

- Any restrictions to use by non-academics: Contact authors

- Manuscript data: https://github.com/Niema-Lab/ SEPIA-paper-final

\section{Abbreviations}

ART: Antiretroviral therapy; $E_{d}$ : Expected degree; HIV: Human immunodeficiency virus; PLWH: People living with HIV; SEPIA: Simulation-based Evaluation of Prloritization Algorithms; $\lambda_{+}::$Rate of starting ART; $\lambda_{-}=:$Rate of stopping ART.

\section{Acknowledgements}

We would like to thank Vignesh Gokul and Professor Christine Alvarado for their mentorship through the Early Research Scholars Program (ERSP).

\section{Author's contribution}

NM conceived and directed this project. All members wrote the code for this project. KA, TJ, ML, TN, MS and NM composed this manuscript. All authors read and approved the final manuscript.

\section{Funding}

No funding was received for this study. No funding bodies played any role in the design of the study nor in the collection, analysis, and interpretation of data, nor in writing the manuscript.

\section{Declaration}

Consent for publication

Not applicable.
Ethics approval and consent to participate

Not applicable

\section{Competing interests}

The authors declare that they have no competing interests.

Received: 1 March 2021 Accepted: 23 May 2021

Published online: 03 June 2021

References

1. CDC: Prevention. https://www.cdc.gov/hiv/basics/prevention.html (2019-12)

2. Wertheim JO, Murrell B, Mehta SR, Forgione LA, Kosakovsky Pond SL, Smith DM, Torian LV. Growth of hiv-1 molecular transmission clusters in New York City. J Infect Dis. 2018;218(12):1943-53. https://doi.org/10.1093/ infdis/jiy431.

3. Pond SLK, Weaver S, Brown AJL, Wertheim JO. HIV-trace (transmission cluster engine): a tool for large scale molecular epidemiology of HIV-1 and other rapidly evolving pathogens. Mol Biol Evol. 2018;35(7):1812-9. https://doi.org/10.1093/molbev/msy016.

4. Moshiri N, Smith DM, Siavash M. HIV care prioritization using phylogenetic branch length. J AIDS. 2021:86(5):626-37. https://doi.org/10.1097/ QAl.0000000000002612.

5. Moshiri N, Ragonnet-Cronin M, Wertheim JO, Mirarab S. Favites: simultaneous simulation of transmission networks, phylogenetic trees and sequences. Bioinformatics. 2018;35(11):1852-61. https://doi.org/10.1093/ bioinformatics/bty921.

6. Ratmann O, Hodcroft EB, Pickles M, Cori A, Hall M, Lycett S, Colijn C, Dearlove B, Didelot X, Frost S, et al. Phylogenetic tools for generalized HIV-1 epidemics: findings from the Pangea-HIV methods comparison. Mol Biol Evol. 2016:34(1):185-203. https://doi.org/10.1093/molbev/msw217.

7. Kendall MG. A new measure of rank correlation. Biometrika. 1938;30(1/2):81-93.

\section{Publisher's Note}

Springer Nature remains neutral with regard to jurisdictional claims in published maps and institutional affiliations.
Ready to submit your research? Choose BMC and benefit from:

- fast, convenient online submission

- thorough peer review by experienced researchers in your field

- rapid publication on acceptance

- support for research data, including large and complex data types

- gold Open Access which fosters wider collaboration and increased citations

- maximum visibility for your research: over 100M website views per year

At BMC, research is always in progress.

Learn more biomedcentral.com/submissions 